\title{
CONTINUOUS TIME SERIES OF WATER VAPOR PROFILES FROM A COMBINATION OF RAMAN LIDAR AND MICROWAVE RADIOMETER
}

\author{
Andreas Foth $^{1}$, Holger Baars ${ }^{2}$, Paolo Di Girolamo ${ }^{3}$, Bernhard Pospichal ${ }^{1}$ \\ ${ }^{1}$ Leipzig Institute for Meteorology (LIM), University of Leipzig, Leipzig, Germany, \\ Email: andreas.foth@uni-leipzig.de \\ ${ }^{2}$ Leibniz Institute for Tropospheric Research (Tropos), Leipzig, Germany \\ ${ }^{3}$ Scuola di Ingegneria, Università degli Studi della Basilicata, Potenza Italy
}

\begin{abstract}
In this paper, we present a method to retrieve continuous water vapor profiles from a combination of a Raman lidar and a microwave radiometer. The integrated water vapor from the microwave radiometer is used to calibrate the Raman lidar operationally resulting in small biases compared to radiosondes. The height limitations for Raman lidars (cloud base and daylight contamination) can be well compensated by the application of a two-step algorithm combining the Raman lidars mass mixing ratio and the microwave radiometers brightness temperatures.
\end{abstract}

\section{INTRODUCTION}

Water vapor plays a key role in the energy budget and in the description of the thermodynamic state. Moreover, it is the most important green house gas. Besides its high spatio-temporal variability, water vapor is involved in many atmospheric processes e.g. cloud formation or the transport of latent heat. This complicates the implementation of water vapor in climate or weather prediction models. To achieve an extensive comprehension spatially and temporally resolved measurements are necessary. In the framework of the German research project High Definition Clouds and Precipitation for advancing Climate Prediction " $\mathrm{HD}(\mathrm{CP})^{2}$ " the intense observation campaign $\mathrm{HD}(\mathrm{CP})^{2}$ Observational Prototype Experiment (HOPE) was performed in western Germany [1]. During HOPE, both active and passive remote sensing systems observed the vertical water vapor distribution. An active method is given by the Raman lidar technique enabling high vertical resolution measurements of water vapor. However, these measurements are highly disturbed by clouds and daylight. Furthermore Raman lidars need to be calibrated with a simultaneously measuring instrument. All these three problems can be compensated by the use of a microwave radiometer (MWR). Passive mi- crowave radiometry provides atmospheric water vapor observations with high temporal, but limited vertical resolution during both day and nighttime. However, the integrated water vapor (IWV) can be retrieved very accurately and can be used to calibrate the Raman lidar. In addition, clouds are semitransparent in the microwave region which allows an operation during all weather conditions except for precipitation.

The synergy of these instruments provides complementary information on the water vapor structure. Thus, when both Raman lidar and MWR are measuring collocated and simultaneously, continuous water vapor profiles can be obtained on a routine basis also during cloudy conditions. This paper has two major objectives which are presented after a brief introduction in the instrumentation and the lidar methodology. Firstly, the application of a calibration method which is only based on IWV from MWR in a very straightforward way to offer a broad application [2]. Secondly, the development of a two-step algorithm combining the Raman lidar mass mixing ratio and the MWR brightness temperature. Applying both steps, the Kalman filter and the optimal estimation method, water vapor from within and above a cloud can be retrieved.

\section{INSTRUMENTATION}

During HOPE ground-based remote sensing observations were conducted around Jülich in western Germany in April and May 2013. Besides the Jülich Observatory for Cloud Evolution (JOYCE) two further observation sites were temporarily set up $4 \mathrm{~km}$ around Jülich, the Leipzig Aerosol and Cloud Remote Observations System (LACROS) and the Karlsruhe Institute for Technologies (KIT) site. The instruments used in this study will be briefly explained in the following subsections. 


\subsection{POLLYXT}

The lidar measurements at the LACROS site were performed with the fully automatic portable multiwavelength Raman and polarization lidar Polly $\mathrm{XT}$ by the Leibniz Institute for Tropospheric Research (TROPOS). The system receives backscattered light at 355, 532 and $1064 \mathrm{~nm}$ and Raman scattered light at 387, 407 and $607 \mathrm{~nm}$ wavelengths. Further informations are given in [3]. No water vapor observations were performed during daytime due to the high daylight background. The temporal and vertical resolutions amount to $30 \mathrm{~s}$ and $30 \mathrm{~m}$, respectively.

\subsection{BASIL}

The University of Basilicata lidar system (BASIL) observations were conducted at the JOYCE site during the HOPE campaign. In contrast to Polly ${ }^{\mathrm{XT}}$ BASIL performs high resolution and accurate measurements of atmospheric water vapor, in both day and nighttime due to its more powerful laser. A thorough description of the technical characteristics, measurement capabilities and performances is given in [4]. For water vapor measurements BASIL uses the same wavelengths as Polly ${ }^{\mathrm{XT}}$. The maximum resolutions are $1 \mathrm{~s}$ in time and $7.5 \mathrm{~m}$ in height, respectively, and can be traded-off to improve the measurement precision.

\subsection{MICROWAVE RADIOMETER HATPRO}

The humidity and temperature profiler (HATPRO) is a passive remote sensing instrument that measures atmospheric emission at two frequency bands in the microwave spectrum. Vertical humidity informations can be retrieved by observing at seven frequencies along the $22.235 \mathrm{GHz} \mathrm{H}_{2} \mathrm{O}$ absorption line. Along the $\mathrm{O}_{2}$ absorption complex from 51 to $58 \mathrm{GHz}$ vertical temperature informations can be retrieved. To obtain atmospheric quantities such as IWV, statistical retrievals were used. Such retrievals are based on a multi-linear regression between atmospheric profiles and modeled brightness temperatures. Both MWRs from JOYCE and LACROS utilize the same retrieval algorithms based on a long term dataset of radiosondes.

\section{AUTOMATED CALIBRATION}

The following section dealing with the calibration of the Raman lidar mass-mixing ratio is based on [2]. Using the inelastic backscatter from molecular nitrogen $\left(\mathrm{N}_{2}\right)$ at $387 \mathrm{~nm}$ and from water vapor $\left(\mathrm{H}_{2} \mathrm{O}\right)$ at $407 \mathrm{~nm}$, the Raman lidar technique enables the determination of water vapor mixing ratio profiles [5]. The mass mixing ratio can be obtained by forming

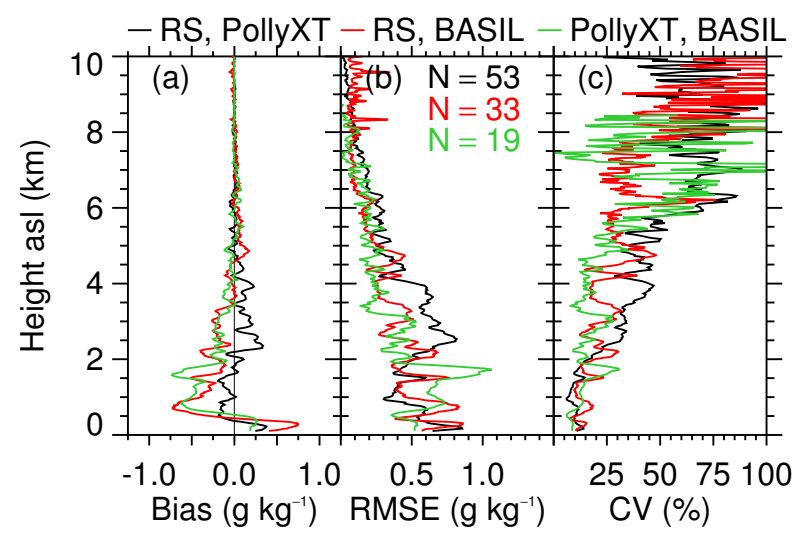

Figure 1: Figure 8. (a) Absolute bias between the radiosonde (RS) and Polly ${ }^{\mathrm{XT}}$ (black), between RS and BASIL (red) and between Polly ${ }^{\mathrm{XT}}$ and BASIL (green). (b) Root-mean-square error (RMSE) of the water vapour mixing ratio. The numbers indicate the sample size. (c) Coefficient of variation ( $\mathrm{CV}$, relative RMSE).

and rearranging the ratio of the Raman lidar equations for both signals:

$$
m_{\mathrm{H}_{2} \mathrm{O}}(z)=C \frac{P_{\lambda_{\mathrm{H}_{2} \mathrm{O}}}(z)}{P_{\lambda_{\mathrm{N}_{2}}}(z)} \frac{T_{\lambda_{\mathrm{N}_{2}}}(z)}{T_{\lambda_{\mathrm{H}_{2} \mathrm{O}}}(z)} .
$$

In Eq. (1) identical overlap factors were assumed. The last term in Eq. (1) describes the differential transmission ratio at $\lambda_{\mathrm{N}_{2}}$ and $\lambda_{\mathrm{H}_{2} \mathrm{O}}$ which is not explained in detail here. The second term indicates the signal ratio which is directly measured. Differences in the range-independent Raman backscatter cross sections for both channels are adsorbed within the calibration factor $C$ which can be determined by comparison with a simultaneous measurement from a reference instrument. Usually, a linear regression between the water vapor mixing ratio from a radiosonde and the signal ratio $P_{\mathrm{H}_{2} \mathrm{O}} / P_{\mathrm{N}_{2}}$ from the lidar is used [6] and the calibration factor is defined as the slope of the regression line. However, in previous experiments radiosondes showed a significant sonde-to-sonde variability [7] as well as a dry bias [8]. Therefore, Raman lidars are often calibrated by using the IWV retrieved from a MWR. Using this method $C$ is defined as the ratio between the IWV measured with the MWR and the integrated signal ratio from the lidar. $C$ can be calculated from the mean of each 20 min clear sky interval.

The calibration factor from Polly ${ }^{\mathrm{XT}}$ based on the IWV from MWR varies only slightly with a relative error of $5 \%$ during the two month of HOPE. Such high stability of the calibration factor allows deriving Raman lidar water vapor profiles also dur- 
ing cloudy conditions. In these cases the calibration factor from the last 20 min clear sky interval can be applied. With this technique continuous water vapor profiles up to cloud base can be retrieved during all non-precipitating night cases. A statistical analysis of the absolute bias, the root mean square error (RMSE) and the coefficient of variation $(\mathrm{CV})$ between the radiosonde and the two lidars is given in Fig. 1. For the comparisons with the radiosonde only clear sky nighttime measurements within less than $2 \mathrm{~h}$ before or after the radiosonde launch time are considered. The profiles are averaged over $20 \mathrm{~min}$ and are interpolated to the lidars height grid. For the comparison between both lidars only simultaneous 20 min averages are investigated. One has to consider that several lidar profiles were compared to one RS profile (e.g. lidar profiles from 21:20, 21:40 and 22:00 UTC to the radiosonde from 23:00 UTC). The biases are largest in the planetary boundary layer with maximum values values of about $0.75 \mathrm{~g} \mathrm{~kg}^{-1}$ mostly induced by inhomogeneous conditions at the different measurement locations (distances about $4 \mathrm{~km}$ ). Above the planetary boundary layer the bias converges to zero. The RMSE decreases with height and the CV increases with height due to the decreasing amount of water vapor. Finally, these low biases confirm the application of the MWR to calibrate the Raman lidar resulting in rather accurate profiles.

\section{RETRIEVAL}

After automatically calibrating the lidar measurement, the two-step algorithm is applied which is sketched in Fig. 2. If the calibrated profiles are truncated at the cloud base (determined from the lidar $1064 \mathrm{~nm}$ channel) they need to be expanded to the full height range. This is done by the Kalman filter [10] using a combination of the current calibrated lidar measurement and an estimated state which is the time projection of a previous analyzed state. The resulting filtered state serves as input to the one-dimensional variational (1D-VAR) algorithm. The optimum estimation of the atmospheric state by a given MWR measurement of brightness temperatures $(\mathbf{z})$ and the filtered state as a priori $\left(\mathrm{x}^{\mathrm{F}}\right)$ can be found by minimizing a cost function of the form [10]:

$$
\begin{aligned}
\mathrm{J}(\hat{\mathbf{x}}) & =\left[\hat{\mathbf{x}}-\mathbf{x}^{\mathrm{F}}\right]^{T} \mathbf{P}^{\mathrm{F}}\left[\hat{\mathbf{x}}-\mathbf{x}^{\mathrm{F}}\right] \\
& +[\mathbf{z}-\mathbf{F}(\hat{\mathbf{x}})]^{T} \mathbf{P}_{\mathrm{z}}^{-1}[\mathbf{z}-\mathbf{F}(\hat{\mathbf{x}})]+\mathbf{J}_{\mathrm{p}}
\end{aligned}
$$

The time index $k$ is omitted here for clarity. $\hat{\mathbf{x}}$ is the optimal estimate of the atmospheric state. $\mathbf{P}^{\mathrm{F}}$ and $\mathbf{P}_{\mathrm{z}}$ denote the error covariance matrices of the filtered state and the MWR measurement, respectively. $\mathbf{J}_{\mathrm{p}}$ indicates a penalty term to avoid supersaturation. The forward model operator $\mathbf{F}(\mathbf{x})$ calculates the brightness temperatures from a given state $\mathbf{x}$. For the modeling of the brightness temperatures from a given profile a non-scattering microwave radiative transfer model is used for gas absorption [11] and liquid water absorption [12]. For the calculation of liquid water a modified adiabatic assumption is used [13]. $\mathrm{J}(\hat{\mathbf{x}})$ is minimized iteratively using the Levenberg-Marquardt method [10]. The iteration starts with a first guess which is based on the average of 225 radiosonde profiles (during HOPE) and stops after the convergence criterion is reached. In summary, the filtered profiles are modified such that the modeled brightness temperatures match those measured with the MWR. Figure 3 illustrates the retrieved mixing ratio profiles (a) and associated errors (b) from a Polly ${ }^{\mathrm{XT}}$ observation on 22 April 2013. The time resolution amounts to $5 \mathrm{~min}$. The solid white line indicates the truncation height. While only using the lidar no water vapor observation from above the truncation height is available due to daylight contamination between 18:00 and about 19:30 UTC and strong signal attenuation within clouds (dotted white line). But in

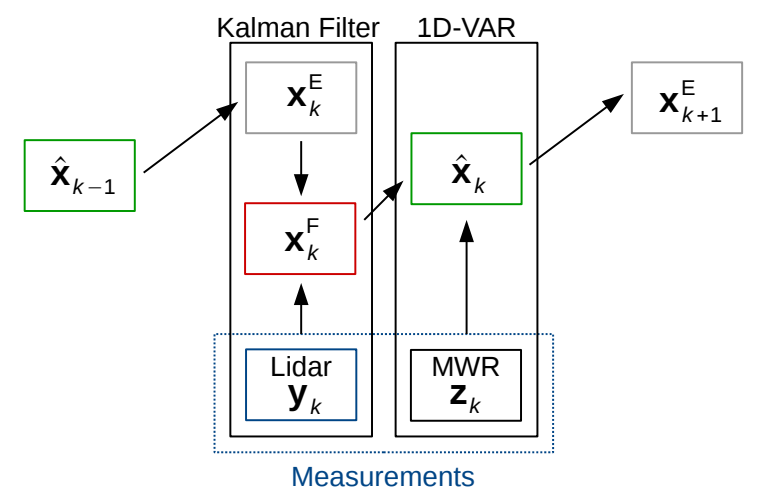

Figure 2: Sketch of the retrieval scheme. Starting with an analyzed state $\hat{\mathbf{x}}_{k-1}$ which is projected in time to the estimated state $\mathbf{x}_{k}^{\mathrm{E}}$. This state is then combined with the current lidar measurement $\mathbf{y}_{k}$ to the filtered state $\mathbf{x}_{k}^{\mathrm{F}}$ using the Kalman filter. $\mathbf{x}_{k}^{\mathrm{F}}$ is then used as the a priori input to the one-dimensional variational (1D-VAR) assimilation scheme. The a priori profile is modified such that the modeled brightness temperature match those measured with the microwave radiometer $(\mathrm{MWR}) \mathbf{z}_{k}$ resulting in the most probable estimated state $\hat{\mathbf{x}}_{k}$ which is again projected in time in the consecutive step. This figure is adapted from [9]. 


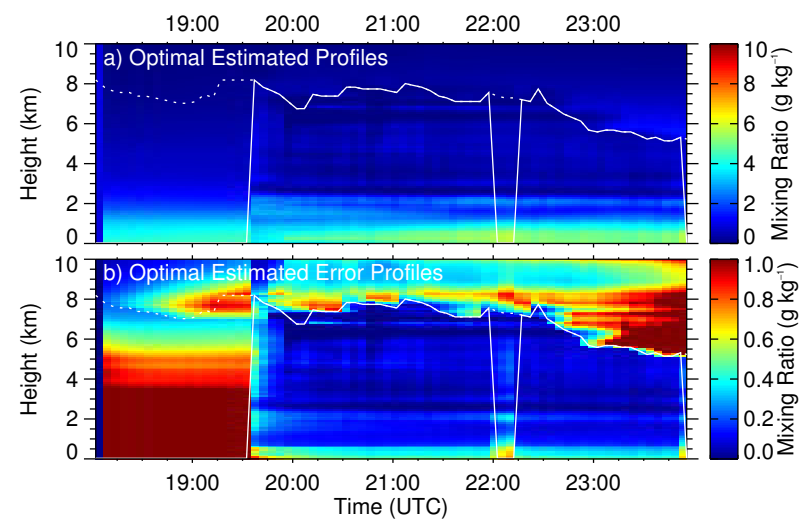

Figure 3: (a) Height-time display of the optimal estimated mixing ratio profiles derived from Polly ${ }^{\mathrm{XT}}$ observations on 22 April 2013. (b) Associated Errors. The solid white line indicates the truncation height and the dotted line indicates the cloud base.

combination with a microwave radiometer and by applying the two-step algorithm water vapor information can also be retrieved from within or above the cloud and during strong daylight contamination. Between 18:00 and 19:30 UTC the retrieved profiles are driven by the first guess. Therefore, no precise layers can be retrieved. At around 22 UTC the profiles are truncated due to the routine depolarization calibration of the lidar. The larger errors near the surface are caused by the manual increase of the lidar measurement error to account for the overlap problem. Above the cloud base an enhanced a priori error is applied.

\section{OUTLOOK}

With the presented automatic algorithm continuous water vapor profiles can be retrieved during all nonprecipitating conditions on a routine basis but with limited maximum lidar measurement height during daytime. In the next steps we will evaluate the retrieval with radiosonde launches. We plan to apply the retrieval to the data sets of HOPE and LACROS in Leipzig. This technique in combination with Doppler lidar data enables the investigation of turbulent entrainment of water vapor at cloud boundaries. In the future this algorithm can be implemented in the Cloudnet data processing [14].

\section{REFERENCES}

[1] Macke, A. 2014, HOPE-A German intensive field campaign to capture the spatiotemporal variability of the thermodynamics, energetics, and microphysics of the cloudy troposphere with high resolution, 14th Conference on Atmospheric Radiation, J6.4, Boston, MA, USA, July 07 - 11, 2014.

[2] Foth, A. et al. 2015, Water vapour profiles from Raman lidar automatically calibrated by microwave radiometer data during HOPE, Atmos. Chem. Phys. Discuss. 15, 6567-6599.

[3] Althausen, D. et al. 2009, Portable Raman lidar PollyXT for automated profiling of aerosol backscatter, extinction, and depolarization, $J$. Atmos. Oceanic Technol. 26, 2366-2378.

[4] Di Girolamo, P. et al. 2009, Multiparameter Raman lidar measurements for the characterization of a dry stratospheric intrusion event, $J$. Atmos. Oceanic Technol. 26, 1742-1762.

[5] Whiteman, D. N. 2003, Examination of the traditional Raman lidar technique. II. Evaluating the ratios for water vapor and aerosols, Appl. Opt. 42, 2593-2608.

[6] England, M. N. et al. 1992, Atmospheric water vapor measurements: Comparison of microwave radiometry and lidar, J. Geophys. Res. 97, 899-916.

[7] Nash, J. et al. 2005, WMO intercomparison of high quality radiosonde systems: Final report, WMO Rep. p. 118 pp.

[8] Turner, D. D. et al. 2003, Dry Bias and Variability in Vaisala RS80-H Radiosondes: The ARM Experience, J. Atmos. Oceanic Technol. 20, 117-132.

[9] Schneebeli, M. 2009, Advancements in Ground-Based Microwave Remote Sensing of the Troposphere - Calibration, Data Retrieval and Applications, $\mathrm{PhD}$ thesis, Institute of Applied Physics, University of Bern.

[10] Rodgers, C. D. 2000, Inverse Methods for Atmospheric Sounding - Theory and Practice, 2, Wolrd Scientific Publishing.

[11] Rosenkranz, P. W. 1998, Water vapor microwave continuum absorption: A comparison of measurements and models, Radio Science 33, 919-928.

[12] Liebe, H. J. et al. 1991, A model for the complex permittivity of water at frequencies below $1 \mathrm{THz}$, I. J. Infrared Milli. 12, 659-675.

[13] Karstens, U. et al. 1994, Remote sensing of cloud liquid water, Meteorol. Atmos. Phys. 54, 157-171.

[14] Illingworth A. J. et al. 2007, Cloudnet, Bull. Amer. Meteorol. Soc. 88, 883-898. 